\title{
Dishabituation in Aplysia can involve either reversal of habituation or superimposed sensitization
}

\author{
Robert D. Hawkins, ${ }^{1,2,4}$ Tracey E. Cohen, ${ }^{1}$ and Eric R. Kandel ${ }^{1,2,3}$ \\ ${ }^{1}$ Center for Neurobiology and Behavior, Columbia University, New York, New York 10032, USA; ${ }^{2}$ New York State Psychiatric \\ Institute, New York, New York 10032, USA; ${ }^{3}$ Howard Hughes Medical Institute, New York, New York 10032, USA
}

\begin{abstract}
Dishabituation has been thought to be due either to reversal of the process of habituation or to a second process equivalent to sensitization superimposed on habituation. One way to address this question is by testing whether dishabituation and sensitization can be dissociated. Previous studies using this approach in Aplysia have come to different conclusions about the nature of dishabituation, perhaps because those studies differed in many respects, including (1) whether they also observed transient behavioral inhibition, and (2) whether they used test stimuli that activated the LE siphon sensory neurons or as yet unidentified sensory neurons. To attempt to resolve the apparent contradictions between the previous studies, we have explored the importance of these two factors by performing a parametric study of dishabituation and sensitization of gill withdrawal in a simplified preparation that does not exhibit transient behavioral inhibition, using two different test stimuli that are known to activate the LE (Touch) or unidentified (Not Touch) sensory neurons. We find that dishabituation and sensitization in this preparation have similar time courses and generally similar functions of shock intensity. However, under one condition, with the Not Touch stimulus $2.5 \mathrm{~min}$ after the shock, dishabituation has a reverse effect of shock intensity. Additional analyses suggest that dishabituation with the Not Touch stimulus 2.5 min after the shock is due to reversal of habituation, whereas $12.5 \mathrm{~min}$ after the shock, dishabituation is due to superimposed sensitization. These results thus suggest that dishabituation may involve either process in the same preparation, and begin to define the conditions that favor one or the other.
\end{abstract}

Historically, there have been two schools of thought about the nature of dishabituation. Pavlov (1927) thought that dishabituation was due to reversal of the process of habituation, whereas Grether (1938) proposed that dishabituation was a second process equivalent to sensitization that was superimposed on habituation. Studies of the cat leg flexion reflex (Spencer et al. 1966; Groves and Thompson 1970) and the leech shortening reflex (Ehrlich et al. 1992; Sahley et al. 1994) have provided experimental evidence for this "dual process" theory, but similar studies of the Aplysia gill- and siphon-withdrawal reflex have produced mixed results. For example, some studies have found that the same noxious stimulation produces both dishabituation and sensitization with similar time courses, suggesting that these forms of learning may involve similar processes (Carew et al. 1971; Cohen et al. 1997; Antonov et al. 1999). By contrast, other studies have found that noxious stimulation produces dishabituation with a lower threshold and faster onset than sensitization, suggesting that they might involve different processes (Marcus et al. 1988). Moreover, studies of cellular analogs of dishabituation and sensitization have also suggested that although synaptic facilitation appears similar in the two cases, it involves different molecular mechanisms (Gingrich and Byrne 1985; Hochner et al. 1986; Ghirardi et al. 1992). However, whether those differences are expressed at the behavioral level is not known.

The mixed results in the behavioral studies may be due in part to the fact that the different studies used different preparations, protocols, test stimuli, response measures, and noxious (dishabituating/sensitizing) stimuli (Hawkins et al. 1998). In addition, some of the differences in results might be explained by two unexpected observations that were made during these stud-

\footnotetext{
${ }^{4}$ Corresponding author.

E-mail rdh1@columbia.edu; fax (212) 543-5474.

Article published online before print. Article and publication date are at http:// www.learnmem.org/cgi/doi/10.1101/lm.49706.
}

ies. First, under some conditions sensitization is preceded by transient ( $<5 \mathrm{~min}$ ) behavioral inhibition, whereas dishabituation is not (Mackey et al. 1987; Marcus et al. 1988). Hawkins et al. (1998) found that the inhibition occurs under the same conditions in which there is a difference between the time courses of dishabituation and sensitization, suggesting that the behavioral differences between dishabituation and sensitization may be due to this additional process of inhibition. Second, during the course of cellular studies of dishabituation and sensitization, it became apparent that there exists a second, as yet unidentified population of siphon sensory neurons with lower thresholds and shorter latencies than the known LE sensory neurons (Frost et al. 1997). These unidentified sensory neurons can be activated by water movement stimulation, whereas the LE sensory neurons are activated only when the skin is touched, and different behavioral studies used test stimuli that would have activated the different populations. Because very little is known about the plasticity of the unidentified sensory neurons, it is possible that dishabituation and sensitization might have different properties depending on which sensory neurons are activated.

To attempt to reconcile the mixed results from previous studies, we have explored the importance of these two factors by performing a parametric study of dishabituation and sensitization in a semi-intact preparation that was initially used to characterize the two populations of sensory neurons, using two different stimuli (Touch and Not Touch) that are known to activate the different populations (Frost et al. 1997). Cohen et al. (1997) examined dishabituation and sensitization with the Touch stimulus in this preparation, and found that they have similar time courses, with no transient behavioral inhibition, but did not investigate whether they also have similar shock thresholds. We have therefore extended their protocol by using three different levels of shock as well. Our results confirm that dishabituation and sensitization have similar time courses, and show that they 
also have similar functions of shock intensity both with the Touch stimulus and with the Not Touch stimulus 12.5 min after the shock. However, under one condition, with the Not Touch stimulus $2.5 \mathrm{~min}$ after the shock, stronger shock was more effective for sensitization but less effective for dishabituation. Additional analyses suggest that dishabituation with the Not Touch stimulus $2.5 \mathrm{~min}$ after the shock is due to reversal of the process of habituation, whereas $12.5 \mathrm{~min}$ after the shock dishabituation is due to superimposed sensitization.

\section{Results}

\section{Dishabituation differs parametrically from sensitization under one condition: With the Not Touch stimulus 2.5 min after the shock}

We performed a parametric study of dishabituation and sensitization of the Aplysia gill-withdrawal reflex in a simplified preparation previously described by Cohen et al. (1997) (Fig. 1), examining the effects of four experimental variables: whether the preparation was first habituated (sensitization vs. dishabituation), whether the tapper touched the siphon (Touch vs. Not Touch), time of testing after the mantle shock (2.5 min vs. 12.5 $\mathrm{min})$, and shock intensity $(1 \times 25 \mathrm{~mA}, 4 \times 25 \mathrm{~mA}$, or $4 \times 100$ $\mathrm{mA})$ (Fig. 2). There were significant main effects of time $\left(F_{[1,288]}=31.26, P<0.001\right)$ and shock $\left(F_{[2,288]}=5.34, P<0.01\right)$ in a four-way ANCOVA with the pre-test as a covariate. Taking the pre-test levels into account, there were not significant main effects of either Sensitization vs. Dishabituation or Touch vs. Not Touch. However, there were several significant interactions involving those factors, including Sen/Dis $\times$ Touch/Not Touch $\times$ Time $\left(F_{[1,288]}=12.58, P<0.001\right)$ and Sen $/$ Dis $\times$ Time $\times$ Shock $\left(F_{[2,288]}=4.14, P<0.05\right)$. To elucidate the meaning of those interactions, we analyzed different subsets of the data separately.

The sensitization data (Fig. 2A) show a simple pattern: There was a significant main effect of time $\left(F_{[1,123]}=6.08, P<0.05\right)$, as in the overall analysis, with a greater increase in the gill- withdrawal reflex 12.5 min than 2.5 min after the shock. There was also a significant main effect of shock $\left(F_{[2,123]}=3.88\right.$, $P<0.05)$, with the stronger shock levels being more effective. There was not a significant effect of Touch vs. Not Touch, and there were no significant interactions. Although there was greater sensitization $12.5 \mathrm{~min}$ after the shock, there was also significant sensitization $\left(F_{[1,123]}=40.17, P<0.001\right)$ rather than inhibition 2.5 min after the shock, when inhibition has been observed following tail shock (Mackey et al. 1987; Hawkins et al. 1998). Because inhibition is sometimes more apparent with shorter delays (Mackey et al. 1987; Hawkins et al. 1998), we also tested the reflex $30 \mathrm{sec}(0.5 \mathrm{~min})$ after $1 \times 25 \mathrm{~mA}$ shock and still observed significant sensitization $\left(F_{[1,147]}=9.26, P<0.01\right)$. (We were not able to use stronger shocks in these experiments because the gill did not relax within $30 \mathrm{sec}$ ). These results are similar to those of Cohen et al. (1997) in the simplified gillwithdrawal preparation and of Hawkins et al. (1998) in the intact animal, both of whom observed sensitization rather than transient inhibition of the withdrawal reflex following mantle shock.

There was considerable variation in the amount of habituation in the different groups, but on average, there was no habituation with either the Touch or Not Touch stimuli with a 1-h intertrial interval (Fig. 2A, no shock controls). By contrast, five repeated taps at 5-min intervals produced significant habituation with two phases: a sharp drop after the first tap that was somewhat greater with the Not Touch stimulus $\left(F_{[1,780]}=5.30\right.$, $P<0.05$ vs. Touch in a planned comparison following a 2-way ANOVA), followed by a more gradual decline that was approximately exponential (linear on a log scale) and equal with the Touch and Not Touch stimuli (Fig. 3A). The initial difference between the Touch and Not Touch stimuli might be due to a difference in depression of postsynaptic potential (PSPs) from the LE and unknown sensory neurons, but Frost et al. (1997) found that they undergo roughly similar depression. Another possible explanation is that the first Touch stimulus may engage a transient facilitatory process that counteracts the habituation on trial 2 (Hawkins, 1989; Jin and Hawkins 2003). Extrapolating the habituation curves, trial 6 in the 2.5-min dishabituation condition would be expected to be $\sim 15 \%$ lower than trial 5 if there was no shock. The habituation recovered only partially after 15 min of rest (trial 6 in the $12.5 \mathrm{~min}$, no shock condition, $F_{[1,97]}=16.47$, $P<0.01$ vs. trial 1) (Fig. 3B), allowing testing of dishabituation at that time point as well.

Shock produced dishabituation on trial 6 (Post) compared with trial 5 (Pre), with a somewhat different pattern than sensitization (Fig. 2B). There was a significant main effect of time $\left(F_{[1,164]}=29.37, P<0.001\right)$, as in the overall analysis, and two significant interactions involving time: Touch/Not Touch $\times$ Time $\left(F_{[1,164]}=20.67, P<0.001\right)$ and Time $\times$ Shock $\left(F_{[2,164]}=4.04\right.$, $P<0.05)$. These interactions explain the corresponding interactions involving Sen/Dis in the overall analysis and were largely due to the unusual results in the 2.5-min Not Touch condition. The results 12.5 min after the shock were similar to the sensitization results, with no significant difference between Touch and Not Touch (with a tendency for Not Touch to be greater) and a significant ef-
Figure 1. The experimental preparation $(A)$ and behavioral protocol $(B)$. For details, see Materials and Methods. (C) Examples of dishabituation with a test stimulus that either touched or did not touch the siphon 2.5 min after $4 \times 25 \mathrm{~mA}$ mantle shock. $(D)$ Examples of sensitization under similar conditions.
398 Learning \& Memory www.learnmem.org 

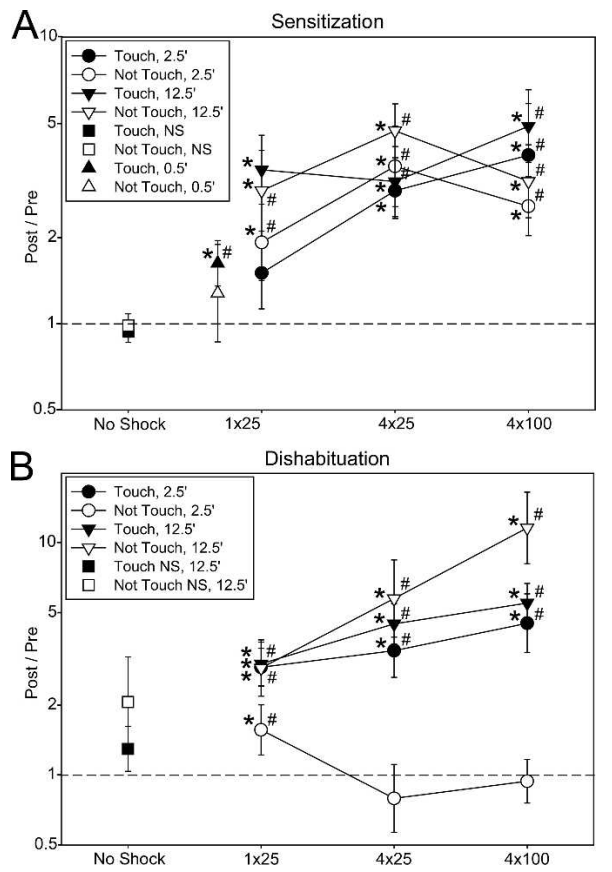

Figure 2. Parametric analysis of sensitization $(A)$ and dishabituation $(B)$ of the gill-withdrawal reflex in response to a stimulus that either touched or did not touch the siphon, tested either $2.5 \mathrm{~min}$ or $12.5 \mathrm{~min}$ after $1 \times 25 \mathrm{~mA}, 4 \times 25 \mathrm{~mA}$, or $4 \times 100 \mathrm{~mA}$ mantle shock, as well as sensitization $30 \mathrm{sec}(0.5 \mathrm{~min})$ after $1 \times 25 \mathrm{~mA}$ shock. The graph shows the least squares mean (corrected for the pre-test amplitude) and SEM Post/ Pre (2/1 for sensitization and $6 / 5$ for dishabituation) in each condition on a logarithmic scale. ${ }^{*} P<0.05$ compared with a Post/Pre ratio of 1 (no change) in planned comparisons following an overall ANCOVA with the pre-test as a covariate. $\# P<0.05$ compared with no shock in one-way ANCOVAs of the Touch and Not Touch data followed by planned comparisons to the internal no shock control data (for sensitization) or the internal habituation control data (for 2.5-min dishabituation), or by Neuman-Keuls post hoc comparisons to 12.5 -min no shock control groups (for 12.5-min dishabituation). The Ns in each group ranged from five to 24 (total, 352).

fect of shock $\left(F_{[2,80]}=6.04, P<0.01\right)$, with increasing shock being more effective. However, $2.5 \mathrm{~min}$ after the shock, there was significantly less dishabituation for Not Touch than for Touch $\left(F_{[1,83]}=16.68, P<0.001\right)$, and this effect was largely due to a reverse effect of shock intensity, with the weakest shock $(1 \times 25$ $\mathrm{mA}$ ) being most effective for Not Touch. There was not a significant difference between Touch and Not Touch for $1 \times 25 \mathrm{~mA}$ shock, but there was a difference for $4 \times 25 \mathrm{~mA}\left(F_{[1,17]}=4.63\right.$, $P<0.05)$ and $4 \times 100 \mathrm{~mA}\left(F_{[1,27]}=8.33, P<0.01\right)$. The Not Touch results are similar to those of Marcus et al. (1988), who used a water movement siphon stimulus in intact animals and found that weak shock was more effective than stronger shock for dishabituation $1.5 \mathrm{~min}$ after the shock.

\section{Dishabituation with the Not Touch stimulus $2.5 \mathrm{~min}$ after the shock may involve reversal of the process of habituation}

These results suggest that dishabituation with the Touch stimulus resembles sensitization both $2.5 \mathrm{~min}$ and $12.5 \mathrm{~min}$ after the shock. By contrast, dishabituation with the Not Touch stimulus may involve different mechanisms at the different times, resembling sensitization $12.5 \mathrm{~min}$ after the shock and perhaps involving reversal of the process of habituation $2.5 \mathrm{~min}$ after the shock. We tested that idea in two additional ways. First, we examined the relationship between the responses on trial 6 and trial 1 , rather than trial 5. If dishabituation is due to reversal of habituation, then the trial 6 response should be smaller than or equal to the trial 1 (unhabituated) response. On the other hand, if dishabituation is due to sensitization superimposed on habituation, then the trial 6 response could be larger or smaller than the trial 1 response. In an ANCOVA of the 6/1 data, there was a significant effect of time $\left(F_{[1,164]}=36.26, P<0.001\right)$ and a significant Touch/ Not Touch $\times$ Time interaction $(F=12.34, P<0.001)$ (Fig. 3B). The trial 6 mean was larger than the trial 1 mean for both the Touch and Not Touch groups $12.5 \mathrm{~min}$ after the shock $(F=21.59$, $P<0.01$ for the Not Touch stimulus). By contrast, the trial 6 mean was smaller than the trial 1 mean for the Not Touch group 2.5 min after the shock $(F=4.83, P<0.05)$ and was not significantly different for the Touch group $2.5 \mathrm{~min}$ after the shock. These results are consistent with the conclusions from the data shown in Figure 2 that dishabituation with either stimulus 12.5 min after the shock may involve superimposed sensitization, whereas with the Not Touch stimulus 2.5 min after the shock, it may involve reversal of habituation.

As another way to test that idea, we examined two correlations, $6 / 5$ vs. $1 / 5$ and $6 / 1$ vs. $5 / 1$. If dishabituation is due to reversal of habituation, then the larger trial 1 is compared with trial 5 (i.e., the larger the habituation), the larger trial 6 should be compared with trial 5 . On the other hand, if dishabituation is due to sensitization superimposed on habituation, then the larger trial 5 is compared with trial 1 (i.e., the smaller the habituation), and the larger trial 6 should be compared with trial 1 . With either the Touch or Not Touch stimulus $2.5 \mathrm{~min}$ after the shock, the $6 / 5$ vs. $1 / 5$ within-groups correlation was larger than the $6 / 1$ vs. $5 / 1$ correlation $\left(t_{[166]}=2.04, P<0.05\right)$, consistent with reversal of habituation (Fig. 3C). With the Not Touch stimulus $12.5 \mathrm{~min}$ after the shock, the $6 / 1$ vs. $5 / 1$ correlation was now larger than the $6 / 5$ vs. $1 / 5$ correlation $\left(t_{[42]}=3.05, P<0.01\right)$, consistent with superimposed sensitization, and with the Touch stimulus $12.5 \mathrm{~min}$ after the shock, the two correlations were not significantly different.

The results of each of these three different analyses suggest that dishabituation with the Not Touch stimulus may involve reversal of habituation $2.5 \mathrm{~min}$ after the shock and superimposed sensitization $12.5 \mathrm{~min}$ after the shock. Results with the Touch stimulus in Figure 3, B and C, are consistent with a similar conclusion, but those in Figure 2 suggest that dishabituation may involve superimposed sensitization at both times. Thus with the Touch stimulus $2.5 \mathrm{~min}$ after the shock, the results are mixed, suggesting that dishabituation may involve a combination of the two mechanisms or additional mechanisms in that case.

\section{Discussion}

These experiments have attempted to resolve the apparent contradictions between previous studies by addressing two questions: (1) Is there any difference at the behavioral level between dishabituation and sensitization that might not be explained by transient inhibition? (2) Do the properties of dishabituation and sensitization depend on whether the siphon stimulus excites the LE sensory cells or the unknown (low threshold) sensory cells?

\section{Behavioral comparisons of dishabituation and sensitization in Aplysia}

Marcus et al. (1988) examined dishabituation and sensitization of the duration of siphon withdrawal by noxious tail stimulation and found several parametric differences between the two forms of learning, suggesting that they involve different processes: (1) dishabituation appeared earlier than sensitization in development; (2) dishabituation was maximal with weak tail stimulation, whereas sensitization was maximal with stronger tail stimu- 

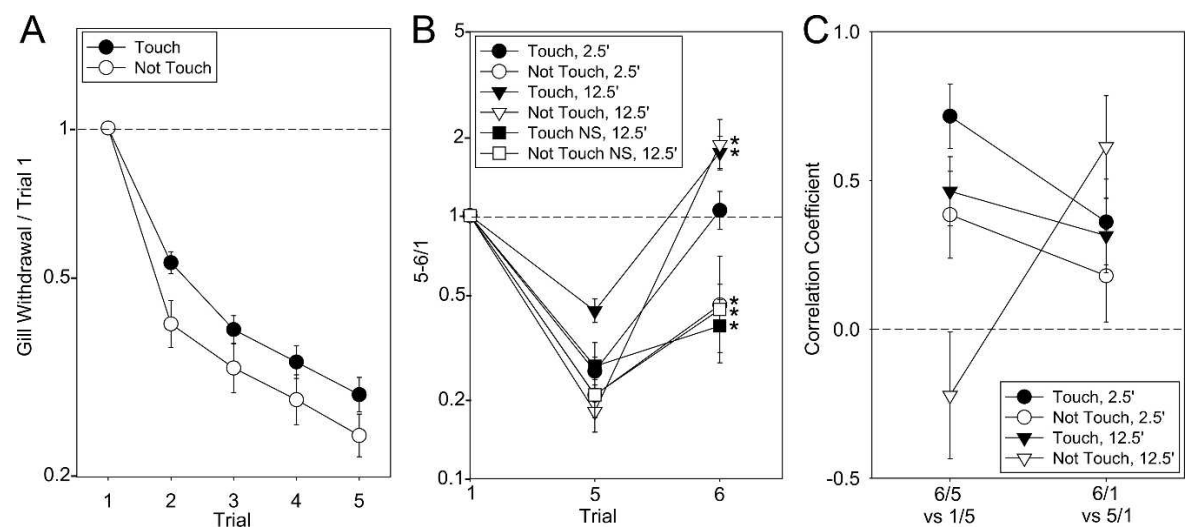

Figure 3. Average habituation and additional tests of whether dishabituation involves reversal of habituation or superimposed sensitization. (A) Mean and SEM habituation on each trial normalized to trial 1 for all experiments with the Touch $(\mathrm{N}=119)$ and Not Touch $(\mathrm{N}=78)$ stimuli. (B) Parametric analysis of dishabituation normalized to trial 1 rather than trial 5 . The graph shows the least squares mean and SEM of each group on the first trial (trial 1), the fifth trial of habituation (trial 5 , the pre-test for dishabituation), and the post-test (trial 6 ). ${ }^{*} P<0.05$ for $6 / 1$ compared with a ratio of 1 (no change) in planned comparisons following ANCOVAs with the pre-test as a covariate. (C) Comparisons of within-groups correlation coefficients expected to reflect either reversal of habituation (trial 6/trial 5 vs. trial 1 /trial 5) or sensitization superimposed on habituation (trial $6 /$ trial 1 vs. trial 5/trial 1 ) for dishabituation with a stimulus that either touched or did not touch the siphon, tested either 2.5 min or 12.5 min after the shock. Data with the three shock intensities have been combined in these figures.

lation; and (3) dishabituation was maximal with a short delay (1.5 $\mathrm{min})$ after the tail stimulation, whereas sensitization was maximal with a longer delay (20 min). However, they could not also test dishabituation with a long delay because of recovery from habituation. In addition, Marcus et al. (1988) and Mackey et al. (1987) found that tail shock produced transient $(<5-\mathrm{min})$ inhibition of the duration of siphon withdrawal with a sensitization design but not with a dishabituation design. By contrast, Cohen et al. (1997) examined dishabituation and sensitization of the amplitude of gill withdrawal by noxious mantle stimulation and found that the same noxious stimulus could produce both effects with similar time courses. They used a relatively long interstimulus interval $(5 \mathrm{~min})$ to produce habituation that recovered slowly, allowing testing of dishabituation with a longer delay. Furthermore, they did not observe transient inhibition following the noxious stimulus, suggesting that the apparent differences between dishabituation and sensitization might be due to differences in a third process, inhibition. However, there were many differences in the experimental conditions and preparations in these studies that might also contribute to the differences in results.

Hawkins et al. (1998) investigated the importance of some of those conditions in the same preparation by comparing dishabituation and sensitization of the amplitude or duration of gill or siphon withdrawal at various times after tail or mantle shock. They observed differences between dishabituation and sensitization only when they measured the duration of siphon withdrawal shortly after tail shock, when there was also transient inhibition with the sensitization design. These results supported the idea that differences between dishabituation and sensitization might be due to differences in inhibition. However, Marcus et al. (1988) used a water movement (Not Touch) stimulus, whereas Cohen et al. (1997) used a Touch stimulus, and Hawkins et al. (1998) did not examine that variable. In addition, only Marcus et al. (1988) varied the intensity of the noxious stimulus. Thus, those factors might also contribute to the differences in results in the different studies.

We have addressed these questions by performing a parametric analysis of dishabituation and sensitization similar to that of Marcus et al. (1988) with the semi-intact preparation and promeasured the amplitude of gill withdrawal at two times $(2.5$ or $12.5 \mathrm{~min})$ after three levels $(1 \times 25,4 \times 25$, or $4 \times 100 \mathrm{~mA}$ ) of mantle shock. In addition, we used two different siphon stimuli: a Touch stimulus that activates the LE siphon sensory cells, and a Not Touch stimulus that does not (Frost et al. 1997). The Not Touch stimulus is qualitatively similar to the water movement stimulus used by Marcus et al. (1988), and the Touch stimulus is qualitatively similar to the tactile stimulus used by Cohen et al. (1997), although the amplitudes and durations of the stimuli were different.

With a sensitization design, we did not observe transient behavioral inhibition either $2.5 \mathrm{~min}$ or $30 \mathrm{sec}$ after the shock, similar to the results of Hawkins et al. (1998) with the same response measure and noxious stimulus in intact animals. However, Cohen et al. (1997) did observe modest inhibition at the cellular level that was outweighed by competing facilitation in this preparation. There was greater sensitization with a longer delay and a stronger shock, and no difference between the Touch and Not Touch stimuli. With a dishabituation design, there was also a larger increase with a longer delay (12.5 $\mathrm{min})$. These results are similar to the behavioral results of Cohen et al. (1997) and support the idea that dishabituation and sensitization have basically similar time courses when they are examined in the absence of transient behavioral inhibition or substantial recovery from habituation. However, with the Not Touch (water movement) stimulus 2.5 min after the shock, dishabituation and sensitization had reverse effects of shock intensity. These results are similar to those of Marcus et al. (1988) and suggest that not all of the differences between dishabituation and sensitization can be explained by transient inhibition. Furthermore, we found that this reverse effect of shock intensity did not occur with the Touch stimulus 2.5 min after the shock or with either stimulus $12.5 \mathrm{~min}$ after the shock, when Marcus et al. (1988) did not test it. Thus our results suggest that dishabituation and sensitization can be dissociated behaviorally, but only under limited conditions: in our experiments with the Not Touch stimulus $2.5 \mathrm{~min}$ after the shock. Consistent with that idea, additional analyses suggested that dishabituation with the Not Touch stimulus involves reversal of the process of habituation $2.5 \mathrm{~min}$ after the shock, whereas dishabituation involves sensitization superimposed on habituation $12.5 \mathrm{~min}$ after the shock. These specific conclusions no doubt depend on the specific preparation, protocol, stimulus intensity and frequency, response measure, etc., that we used. More generally, however, our results suggest that dishabituation may involve either reversal of habituation or superimposed sensitization in the same preparation, and begin to define the types of conditions that favor one process or the other.

\section{Possible implications for cellular mechanisms of dishabituation and sensitization in Aplysia}

Plasticity of the withdrawal reflex is due in part to plasticity at synapses from known (LE) siphon sensory neurons that are activated by tactile stimulation and unknown sensory neurons that are activated by water movement stimulation. Facilitation at syn- 
apses of the LE sensory neurons is thought to involve two different presynaptic mechanisms depending on whether the synapses are rested or depressed, as they would be during sensitization or dishabituation (Byrne and Kandel, 1996), and it may also involve postsynaptic mechanisms under some conditions (Li et al. 2005). Facilitation at rested synapses involves PKA, which produces broadening of presynaptic action potentials. By contrast, facilitation at depressed synapses involves PKC, which may produce mobilization of transmitter vesicles to the readily releasable pool, which is thought to be depleted during depression (Gingrich and Byrne 1985; Hochner et al. 1986; Ghirardi et al. 1992). According to that idea, facilitation at depressed synapses can be thought of as a reversal of the mechanism of depression, and dishabituation would be expected to involve reversal of the process of habituation rather than superimposed sensitization.

However, these different mechanisms of facilitation in vitro may not be reflected at the behavioral level, for two reasons. First, behavioral learning can involve plasticity at several sites in addition to the sensory-motor neuron synapses. Cohen et al. (1997) performed a cellular analysis of dishabituation and sensitization with a Touch stimulus using the same semi-intact preparation as the present study, and found that whereas habituation of the reflex is due primarily to depression of the monosynaptic PSP at sensory-motor neuron synapses, dishabituation and sensitization involve multiple mechanisms, including facilitation of the monosynaptic PSP, facilitation and inhibition of interneurons, and posttetanic potentiation (PTP) at the neuromuscular junction. No one of these mechanisms tracks the behavior, and each contributes somewhat differently under different circumstances. Facilitation of the monosynaptic PSP makes the largest contribution 2.5 min after the shock with a dishabituation design, when the PSP is depressed, whereas plasticity in interneurons makes a relatively larger contribution under the other conditions. Those results suggest that the difference we observed between dishabituation with the Touch and Not Touch stimuli 2.5 min after the shock could be due to differences in facilitation of the monosynaptic PSP from the known (LE) and unknown sensory neurons. Similar to dishabituation with the Touch stimulus, facilitation of depressed PSPs from LE sensory neurons is greater with stronger shock (Wright et al. 1991). Frost et al. (1997) found that PSPs from the unknown sensory neurons undergo depression and facilitation generally similar to those of PSPs from LE sensory neurons, but they did not vary shock intensity. The finding that dishabituation with a Not Touch stimulus has a reverse effect of shock intensity (Fig. 2; Marcus et al. 1988) suggests that facilitation of depressed PSPs from the unknown sensory neurons may also have a reverse effect of shock intensity. Alternatively, dishabituation with a Not Touch stimulus may preferentially involve a covert inhibitory process that is larger with stronger shock. This possibility seems unlikely because all previous studies with Not Touch as well as Touch stimuli have found that inhibition is preferentially involved during sensitization rather than dishabituation (Mackey et al. 1987; Marcus et al. 1988; Wright et al. 1991; Cohen et al. 1997; Hawkins et al. 1998), but both of these possibilities remain to be tested at the cellular level.

Second, even when the monosynaptic PSP makes a large contribution to behavior, the different molecular mechanisms of facilitation in vitro need not appear different at the cellular or behavioral levels. In a semi-intact siphon withdrawal preparation, facilitation of the monosynaptic PSP from LE siphon sensory neurons makes a large contribution and tracks behavioral dishabituation and sensitization (Antonov et al. 1999). In that preparation, as in the gill-withdrawal preparation (Cohen et al. 1997 and Fig. 2), parametric analyses have not revealed any behavioral dissociation between dishabituation and sensitization with a Touch siphon stimulus. Nonetheless, preliminary results from the siphon withdrawal preparation suggest that behavioral sensitization and concurrent facilitation of the monosynaptic PSP importantly involve PKA, whereas dishabituation importantly involves PKC (Antonov et al. 2005). These results are similar to facilitation at rested and depressed synapses of LE sensory neurons in vitro (Ghirardi et al. 1992), and suggest that behavioral dishabituation and sensitization may involve the two different molecular mechanisms of facilitation that have been described in vitro (Gingrich and Byrne, 1985; Hochner et al. 1986). However, those molecular mechanisms give rise to cellular and behavioral phenotypes that are indistinguishable except by pharmacological means. Likewise, other forms of plasticity such as long-term potentiation refer to families of processes that appear similar at the cellular level but can involve different mechanisms at the molecular level depending on factors including the synapses studied, the stage of development, and the induction protocol (see Zalutsky and Nicoll 1990; Huang et al. 1994; Salin et al. 1996; Huang and Kandel 1998; Lev-Ram et al. 2002; Jensen et al. 2003; Yasuda et al. 2003; Zakharenko et al. 2003). These findings thus suggest that mechanisms of plasticity may be "degenerate" in the sense that word is used to describe the genetic code, by which several different DNA sequences may give rise to the same amino acid.

\section{Materials and Methods}

Aplysia californica weighing 85-120 g were obtained from the Howard Hughes Mariculture Facility (Miami, FL). Experiments were performed on a dissected preparation that has been described previously (Cohen et al. 1997) consisting of the siphon, gill, mantle, and abdominal ganglion (Fig. 1A). The behavioral methods were also generally similar to those of Cohen et al. (1997), except that the siphon was stimulated with a tapper driven by a solenoid that pulled against a spring (Frost et al. 1997). The tip of the tapper was soft plastic and $3 \mathrm{~mm}$ in diameter, and the tap duration was $50 \mathrm{msec}$. The force of the taps was adjusted by varying the initial distance between the tip of the tapper and the siphon, with longer distances producing weaker taps. The stimulation was calibrated against a strain gauge transducer (Grass Instruments), and the average pressure when it touched the siphon was $4.1 \mathrm{~g} / \mathrm{mm}^{2}$. In some cases, the tapper was positioned far enough away that it never touched the siphon at all (as judged by positioning the tapper the same distance from the strain gauge), and the stimulus was presumably the water movement produced by the tapper. A silk suture connected the efferent vein of the gill to a low-mass isotonic transducer (Harvard Apparatus), which could reliably record gill withdrawals as small as $\sim 0.1 \mathrm{~mm}$. The peak amplitude of withdrawal was measured by using a laboratory interface to a microcomputer and commercially available software (Hilal Associates) that also controlled the stimulation. During dishabituation and sensitization training, the mantle shelf was stimulated with either a single $60-\mathrm{Hz}$ AC electrical shock ( $1 \mathrm{sec}, 25 \mathrm{~mA}$ ) or a train of four shocks (2-sec interstimulus interval (ISI), 25 or $100 \mathrm{~mA}$ ) delivered via fixed electrodes. The average gill withdrawals produced by the three levels of shock were 10.1, 12.5, and $12.2 \mathrm{~mm}$ $\left(F_{[2,287]}=29.50, P<0.001\right)$. Experiments were continued only if the response to the first siphon stimulation (trial 1) was between 0.2 and $10 \mathrm{~mm}$ and the response to the mantle shock was at least $6 \mathrm{~mm}$.

The preparation was rested for at least $1 \mathrm{~h}$ before the beginning of dishabituation or sensitization training (Fig. 1B-D). During habituation, the siphon was stimulated five times with a 5-min intertrial interval, and habituation was measured as the decrease in responding on trial 5 compared with trial $1(5 / 1)$. The mantle was then shocked 2.5 min after trial 5, and the siphon was stimulated again (trial 6) either 2.5 or $12.5 \mathrm{~min}$ after the shock (5 or 15 min after trial 5). Dishabituation was measured as the increase in responding on trial 6 compared with trial $5(6 / 5)$. The habituation parameters in this study are the same as those used by Cohen et al. (1997) and are within the range of other 
previous studies of dishabituation, which have typically used four to 20 stimuli with an interstimulus interval of $30 \mathrm{sec}$ to 5 min (Kupfermann et al. 1970; Pinsker et al. 1970; Carew et al. 1971; Mackey et al. 1987; Marcus et al. 1988; Glanzman et al. 1989; Wright et al. 1991; Hawkins et al. 1998; Antonov et al. 1999). We used a 5-min ISI because it produces slower recovery from habituation, allowing measurement of the time course of dishabituation. During sensitization, there was a single siphon stimulation (trial 0), a 1-h rest followed by a second siphon stimulation (trial 1) to test for habituation with that interstimulus interval, and another 1-h rest. The mantle was then shocked, and the siphon was stimulated again (trial 2) either $2.5 \mathrm{~min}$ or 12.5 min after the shock. Sensitization was measured as the increase in responding on trial 2 compared with trial $1(2 / 1)$.

Because the Post/Pre measure for dishabituation and sensitization was skewed toward large values and therefore highly non-normal $\left(\chi^{2}=620.76, \mathrm{df}=7, P<0.001\right)$, we used a log transformation, which made the distribution of the results approximately normal overall $\left(\chi^{2}=17.24, \mathrm{df}=11\right.$, not significant) (Fig. $4 \mathrm{~A}$ ) and for each of the major subgroups (data not shown). The graphs show the corresponding geometric means on a log scale. The Post/Pre scores did not correlate significantly with the shock responses, but they did correlate negatively with the Pre value overall (within groups $r=-0.51, t_{[288]}=10.16, P<0.001$ ) (Fig. $4 \mathrm{~B}$ ) with similar slopes for each of the major subgroups (data not shown), presumably because of a ceiling effect. This effect would tend to decrease disproportionately the Post/Pre scores of the sensitization and Touch groups, which on average had higher pre-test scores than the dishabituation and Not Touch groups (sen vs. dis, $F_{[1,309]}=145.67, P<0.001$; Touch vs. Not Touch, $F=132.77, P<0.001$ ) (Fig. 4C). In addition, differences between the Touch and Not Touch stimuli were confounded with differences in stimulus strength and thus Pre values, although there was considerable overlap between the groups (Fig. 4B). We addressed these problems by using the pre-test score as a covariate in three- or four-way ANCOVAs, which should give estimates of the effects of Sen/Dis and Touch/Not Touch independent of differences in pre-test values or stimulus strength. We used the
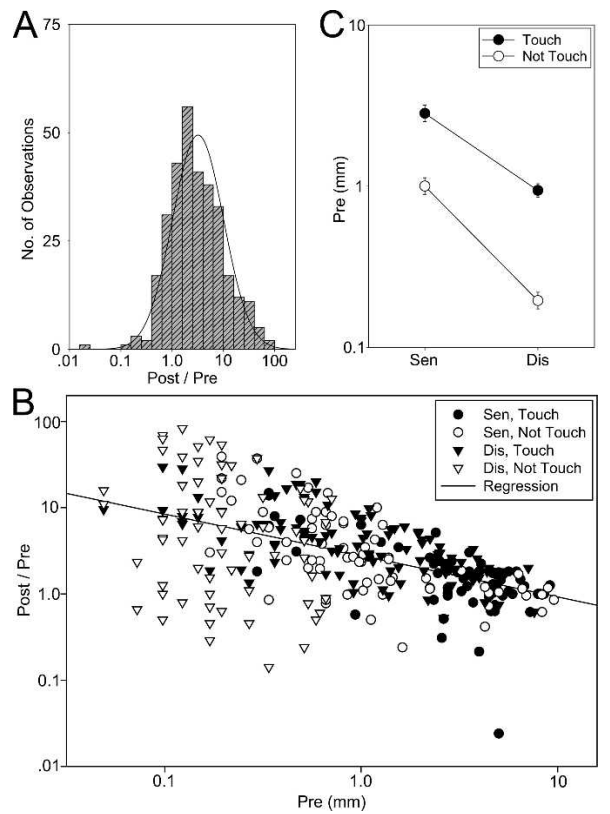

Figure 4. Statistical properties of the behavioral response measure. (A) The Post/Pre measure of dishabituation (6/5) and sensitization $(2 / 1)$ is approximately normally distributed (solid curve) on a logarithmic scale. $(B)$ The Post/Pre measure is negatively correlated with the Pre value (the solid line is the linear regression for all of the data plotted on a log-log scale). (C) The average Pre values were larger for sensitization than for dishabituation and for the Touch stimulus than the Not Touch stimulus.
ANCOVAs to test for main effects and interactions of the experimental variables, followed by planned comparisons of the group means to a Post/Pre ratio of 1 (no change). We also ran two additional sensitization groups (0.5-min Touch and Not Touch) and analyzed them similarly with one-way ANCOVAs. In addition, we compared each of the sensitization groups to no shock by subtracting the animals' internal no shock controls (trial 1/0) from their sensitization scores $(2 / 1)$ and performing one-way ANCOVAs for the Touch and Not Touch data, followed by planned comparisons of the group means to zero (no difference from the internal control). We performed a similar analysis for the 2.5-min dishabituation data, subtracting the animals' internal habituation controls (trial 5/4) from their dishabituation scores (trial 6/5). Because we expected some recovery from habituation in the 12.5-min dishabituation groups, we also ran no shock controls for those groups with the Touch and Not Touch stimuli, and compared each of the other 12.5 min dishabituation groups to the corresponding control group in one-way ANCOVAs followed by Neuman-Keuls post hoc tests. Animals were randomly assigned to the different groups.

\section{Acknowledgments}

This research was supported by grants from the NIH (MH26212) and Howard Hughes Medical Institute.

\section{References}

Antonov, I., Kandel, E.R., and Hawkins, R.D. 1999. The contribution of facilitation of monosynaptic PSPs to dishabituation and sensitization of the Aplysia siphon withdrawal reflex. J. Neurosci. 19: $10438-10450$

. 2005. Roles of PKA, PKC, and CamKII in dishabituation and sensitization of the Aplysia siphon-withdrawal reflex. Abstract Viewer/Itinerary Planner, Program No. 540.6. Society for Neuroscience, Washington, DC.

Byrne, J.H. and Kandel, E.R. 1996. Presynaptic facilitation revisited: State and time dependence. J. Neurosci. 16: 425-435.

Carew, T.J., Castellucci, V.F., and Kandel, E.R. 1971. An analysis of dishabituation and sensitization of the gill-withdrawal reflex in Aplysia. Int. J. Neurosci. 2: 79-98.

Cohen, T.E., Kaplan, S.W., Kandel, E.R., and Hawkins, R.D. 1997. A simplified preparation for relating cellular events to behavior: Mechanisms contributing to habituation, dishabituation, and sensitization of the Aplysia gill-withdrawal reflex. J. Neurosci. 17: 2886-2899.

Ehrlich, J.S., Boulis, N.M., Karrer, T., and Sahley, C.L. 1992. Differential effects of serotonin depletion on sensitization and dishabituation in the leech, Hirudo medicinalis. J. Neurobiol. 23: 270-279.

Frost, L., Kaplan, S.W., Cohen, T.E., Henzi, V., Kandel, E.R., and Hawkins, R.D. 1997. A simplified preparation for relating cellular events to behavior: Contribution of LE and unidentified siphon sensory neurons to mediation and habituation of the Aplysia gilland siphon withdrawal reflex. J. Neurosci. 17: 2900-2913.

Ghirardi, M., Braha, O., Hochner, B., Montarolo, P.G., Kandel, E.R., and Dale, N. 1992. Roles of PKA and PKC in facilitation of evoked and spontaneous transmitter release at depressed and nondepressed synapses in Aplysia sensory neurons. Neuron 9: 479-489.

Gingrich, K.J. and Byrne, J.H. 1985. Simulation of synaptic depression, post-tetanic potentiation, and presynaptic facilitation of synaptic potentials from sensory neurons mediating gill-withdrawal reflex in Aplysia. J. Neurophysiol. 53: 652-659.

Glanzman, D.L., Mackey, S.L., Hawkins, R.D., Dyke, A., Lloyd, P.E., and Kandel, E.R. 1989. Depletion of serotonin in the nervous system of Aplysia reduces the behavioral enhancement of gill withdrawal as well as the heterosynaptic facilitation produced by tail shock. $J$. Neurosci. 9: 4200-4213.

Grether, W.F. 1938. Pseudo-conditioning without paired stimulation encountered in attempting backward conditioning. J. Comp. Psychol. 25: 91-96.

Groves, P.M. and Thompson, R.F. 1970. Habituation: A dual-process theory. Psychol. Rev. 77: 419-450.

Hawkins, R.D. 1989. A biologically based computational model for several simple forms of learning. Psychol. Learn. Motiv. 23: 65-108.

Hawkins, R.D., Cohen, T.E., Greene, W., and Kandel, E.R. 1998. Relationships between dishabituation, sensitization, and inhibition of the gill- and siphon-withdrawal reflex in Aplysia: Effects of response measure, test time, and training stimulus. Behav. Neurosci. 112: 24-38. 
Hochner, B., Klein, M., Schacher, S., and Kandel, E.R. 1986. Additional component in cellular mechanism of presynaptic facilitation contributes to behavioral dishabituation in Aplysia. Proc. Natl. Acad. Sci. 83: 8794-8798.

Huang, Y.Y. and Kandel, E.R. 1998. Postsynaptic induction and PKA-dependent expression of LTP in the lateral amygdala. Neuron 21: $169-178$.

Huang, Y.Y., Li, X.C., and Kandel, E.R. 1994. cAMP contributes to mossy fiber LTP by initiating both a covalently mediated early phase and macromolecular synthesis-dependent late phase. Cell 79: 69-79.

Jensen, V., Kaiser, K.M., Borchardt, T., Adelmann, G., Rozov, A., Burnashev, N., Brix, C., Frotscher, M., Andersen, P., Hvalby, O., et al. 2003. A juvenile form of postsynaptic hippocampal long-term potentiation in mice deficient for the AMPA receptor subunit GluR-A. J. Physiol. 553: 843-856.

Jin, I. and Hawkins, R.D. 2003. Presynaptic and postsynaptic mechanisms of a novel form of homosynaptic potentiation at Aplysia sensory-motor neuron synapses. J. Neurosci. 23: 7288-7297.

Kupfermann, I., Castellucci, V., Pinsker, H., and Kandel, E.R. 1970. Neuronal correlates of habituation and dishabituation of the gill-withdrawal reflex in Aplysia. Science 167: 1743-1745.

Lev-Ram, V., Wong, S.T., Storm, D.R., and Tsien, R.Y. 2002. A new form of cerebellar long-term potentiation is postsynaptic and depends on nitric oxide but not cAMP. Proc. Natl. Acad. Sci. 99: 8389-8393.

Li, Q., Roberts, A.C., and Glanzman, D.L. 2005. Synaptic facilitation and behavioral dishabituation in Aplysia: Dependence on release of $\mathrm{Ca}^{2+}$ from postsynaptic intracellular stores, postsynaptic exocytosis, and modulation of postsynaptic AMPA receptor efficacy. J. Neurosci. 25: $5623-5637$.

Mackey, S.L., Glanzman, D.L., Small, S.A., Dyke, A.M., Kandel, E.R., and Hawkins, R.D. 1987. Tail shock produces inhibition as well as sensitization of the siphon-withdrawal reflex of Aplysia: Possible behavioral role for presynaptic inhibition mediated by the peptide Phe-Met-Arg-Phe-NH ${ }_{2}$. Proc. Natl. Acad. Sci. 84: 8730-8734.

Marcus, E.A., Nolen, T.G., Rankin, C.H., and Carew, T.J. 1988.
Behavioral dissociation of dishabituation, sensitization, and inhibition in Aplysia. Science 241: 210-213.

Pavlov, I.P. 1927. Conditioned reflexes: An investigation of the physiological activity of the cerebral cortex. (trans. G.V. Anrep). Oxford University Press, London.

Pinsker, H., Kupfermann, I., Castellucci, V., and Kandel, E.R. 1970. Habituation and dishabituation of the gill-withdrawal reflex in Aplysia. Science 167: 1740-1742.

Sahley, C.L., Modney, B.K., Boulis, N.M., and Muller, K.J. 1994. The S cell: An interneuron essential for sensitization and full dishabituation of leech shortening. J. Neurosci. 14: 6715-6721.

Salin, P.A., Malenka, R.C., and Nicoll, R.A. 1996. Cyclic AMP mediates a presynaptic form of LTP at cerebellar parallel fiber synapses. Neuron 16: $797-803$.

Spencer, W.A., Thompson, R.F., and Nielson Jr., D.R. 1966. Response decrement of the flexion reflex in the acute spinal cat and transient restoration by strong stimuli. J. Neurophysiol. 29: 221-239.

Wright, W.G., Marcus, E.A., and Carew, T.J. 1991. A cellular analysis of inhibition in the siphon withdrawal reflex of Aplysia. J. Neurosci. 11: $2498-2509$.

Yasuda, H., Barth, A.L., Stellwagen, D., and Malenka, R.C. 2003. A developmental switch in the signaling cascade for LTP induction. Nat. Neurosci. 6: 15-16.

Zakharenko, S.S., Patterson, S.L., Dragatsis, I., Zeitlin, S.O., Siegelbaum, S.A., Kandel, E.R., and Morozov, A. 2003. Presynaptic BDNF required for a presynaptic but not postsynaptic component of LTP at hippocampal CA1-CA3 synapses. Neuron 39: 975-990.

Zalutsky, R.A. and Nicoll, R.A. 1990. Comparison of two forms of long-term potentiation in single hippocampal neurons. Science 248: $1619-1624$.

Received August 4, 2005; accepted in revised form January 27, 2006. 


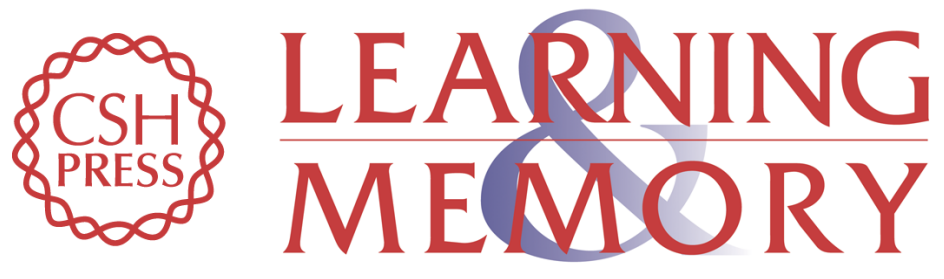

\section{Dishabituation in Aplysia can involve either reversal of habituation or superimposed sensitization}

Robert D. Hawkins, Tracey E. Cohen and Eric R. Kandel

Learn. Mem. 2006, 13:

Access the most recent version at doi:10.1101//m.49706

References This article cites 31 articles, 16 of which can be accessed free at:

http://learnmem.cshlp.org/content/13/3/397.full.html\#ref-list-1

License

Email Alerting Receive free email alerts when new articles cite this article - sign up in the box at the Service top right corner of the article or click here. 